\title{
Article
}

\section{Bounds on the covering radius of some classes of codes over $\mathbb{R}$}

\section{P. Chella Pandian ${ }^{1, *}$}

1 Department of Mathematics, Srimad Andavan Arts and Science College(A), Tiruchirappalli-620005, Tamil Nadu, India.

* Correspondence: chellapandianpc@gmail.com

Received: 22 October 2018; Accepted: 6 January 2019; Published: 24 February 2019.

\begin{abstract}
In this paper, the covering radius of codes over $\mathbb{R}=\mathbb{Z}_{2} R^{*}$, where $R^{*}=\mathbb{Z}_{2}+v \mathbb{Z}_{2}, v^{2}=v$ with different weight are discussed. The block repetition codes over $\mathbb{R}$ is defined and the covering radius for block repetition codes, simplex code of $\alpha$-type and $\beta$-type in $\mathbb{R}$ are obtained.
\end{abstract}

Keywords: Additive codes, covering radius, gray map, Lee weight, Euclidean weight, simplex codes.

MSC: $11 \mathrm{~T} 71,94 \mathrm{~B} 05,11 \mathrm{H} 71$.

\section{Introduction}

C odes over finite commutative rings have been studied for almost 50 years. The main motivation of studying codes over rings is that they can be associated with codes over finite fields through the Gray map. Recently, coding theory over finite commutative non-chain rings is a hot research topic. Recently, there has been substantial interest in the class of additive codes. In [1,2], Delsarte contributes to the algebraic theory of association scheme where the main idea is to characterize the subgroups of the underlying abelian group in a given association scheme.

The covering radius is an important geometric parameter of codes. It not only indicates the maximum error correcting capability of codes, but also relates to some practical problems such as the data compression and transmission. Studying of the covering radius of codes has attracted many coding scientists for almost 30 years. The covering radius of linear codes over binary finite fields was studied in [3].

Additive codes over $\mathbb{Z}_{2} \mathbb{Z}_{4}$ have been extensively studied in [4-7]. Enormous results were made available on the simplex codes over finite fields and finite rings. A few of them are [8-10]. In [11], the authors, in particular, gave lower and upper bounds on the covering radius of codes over the ring $R=\mathbb{Z}_{2}+u \mathbb{Z}_{2}$ where $u^{2}=0$ with respect to different distance, and they explained the covering radius of various repetition codes, Simplex Codes (Type $\alpha$ and $\beta$ over R.) The above results motivate us to work in this area.

In this paper, the simplex codes of types $\alpha$ and $\beta$ over $\mathbb{R}$ are obtained. At this juncture, the meaning of constructing new codes is to concatenate binary and quaternary simplex codes of types $\alpha$ and $\beta$. These results in the simplex codes over $\mathbb{R}$, which contain the corresponding binary and $R^{*}$ codes as subclasses. The rest of this paper is organized as follows

In Section 2, some properties related to additive codes over $\mathbb{R}=\mathbb{Z}_{2} R^{*}$, where $R^{*}=\{0,1, v, 1+v\}, v^{2}=v$ are given. The covering radius of codes over $\mathbb{R}$ is considered in Section 3. In Section 4, the block repetition codes over $\mathbb{R}$ is defined and find their covering radius. The construction of simplex codes over $\mathbb{R}$ of types $\alpha$ and $\beta$ and the covering radius of these codes is also considered in Sections 5.

\section{Preliminaries}

Throughout this paper, $\mathbb{R}=\mathbb{Z}_{2} R^{*}$, where $R^{*}=\{0,1, v, 1+v\}, v^{2}=v$. In this section, some preliminary results are given based on [5] and [7]. A non empty set $C$ is a $\mathbb{R}$-additive code if it is a subgroup of $\mathbb{Z}_{2}^{\gamma} \times R^{*^{\delta}}$. In this case, $C$ is also isomorphic to an abelian structure $\mathbb{Z}_{2}^{\lambda} \times R^{*^{*}}$ for some $\lambda$ and $\mu$. $C$ is of type $2^{\lambda} R^{* \mu}$ as a group. It follows that it has $|C|=2^{\lambda+2 \mu}$ codewords, and the number of order two codewords in $C$ is $|C|=2^{\lambda+\mu}$.

Consider the following extension of the Gray map 


$$
\phi: \mathbb{Z}_{2}^{\gamma} \times R^{*^{\delta}} \rightarrow \mathbb{Z}_{2}^{n} \text {, with } n=\gamma+2 \delta
$$

given by

$$
\phi(u, w)=\left(u, \phi\left(w_{1}\right), \cdots \phi\left(w_{\delta}\right)\right), \forall u \in \mathbb{Z}_{2}^{\gamma}, \forall\left(w_{1}, \cdots, w_{\delta}\right) \in R^{*^{\delta}},
$$

where

$$
\phi: R^{*} \rightarrow \mathbb{Z}_{2}^{2}
$$

is the Gray map given by $\phi(0)=(0,0), \phi(1)=(0,1), \phi(v)=(1,1)$, and $\phi(1+v)=(1,0)$. Then the binary image of a $\mathbb{R}$-additive code under the extended Gray map is called a $\mathbb{R}$-linear code of length $n=\gamma+2 \delta$.

The Hamming weight of $u$, denoted by $w t_{H}(u)$ and $w t_{L}(w)$ and $w t_{E}(w)$ the Lee and Euclidean weights of $w$ respectively, where $u \in \mathbb{Z}_{2}^{\gamma}$ and $w \in R^{*^{\delta}}$ are defined as

$$
w t_{E}\left(v_{i}\right)= \begin{cases}0 & \text { if } v_{i}=0 \\ 1 & \text { if } v_{i}=1 \text { or }(1+v) \\ 4 & \text { if } v_{i}=v\end{cases}
$$

and

$$
w t_{L}\left(v_{i}\right)= \begin{cases}0 & \text { if } v_{i}=0 \\ 1 & \text { if } v_{i}=1 \text { or }(1+v) \\ 2 & \text { if } v_{i}=v\end{cases}
$$

The Lee weight of $x$ is defined as $w t_{L}(x)=w t_{H}(u)+w t_{L}(w)$, where $x=(u, w) \in \mathbb{Z}_{2}^{\gamma} \times R^{*^{\delta}}$, and $u=$ $\left(u_{1}, \cdots, u_{\gamma}\right) \in \mathbb{Z}_{2}^{\gamma}$ and $w=\left(w_{1}, \cdots, w_{\delta}\right) \in R^{*^{\delta}}$ and the Euclidean weight of $x$ is defined as $w t_{E}(x)=$ $w t_{H}(u)+w t_{E}(w)$. The Gray map defined above is an isometry which transforms the Lee distance defined over $\mathbb{Z}_{2}^{\gamma} \times R^{*^{\delta}}$ to the Hamming distance defined over $\mathbb{Z}_{2}^{n}$, with $n=\gamma+2 \delta$.

\section{The covering radius of codes over $\mathbb{R}$}

The covering radius of a code $C$ over $\mathbb{R}$ is introduced in this section. The covering radius of a code $C$ is the smallest number $r$ such that the spheres of radius $r$ around the codewords cover $\mathbb{Z}_{2}^{\gamma} \times R^{*^{\delta}}$. Hence, the covering radius of a code $C$ over $\mathbb{R}$, with respect to the Lee and Euclidean distances is given by

$$
r_{L}(C)=\max _{u \in \mathbb{Z}_{2}^{\gamma} \times R^{* \delta}}\left\{\min _{c \in C} d_{L}(u, c)\right\} \text { and } r_{E}(C)=\max _{u \in \mathbb{Z}_{2}^{\gamma} \times R^{* \delta}}\left\{\min _{c \in C} d_{E}(u, c)\right\}
$$

respectively.

The following result, for codes over $\mathbb{Z}_{4}$, given by Aoki et al. in [12] is also valid for codes over $\mathbb{R}$. Its proof follows from the definition of the covering radius and the fact that the map $\phi$ is a weight preserving map.

Proposition 1. Let $C$ be a code over $\mathbb{Z}_{2}^{\gamma} \times R^{*^{\delta}}$ and $\phi(C)$ be the Gray map image of $C$. Then $r_{L}(C)=r_{H}(\phi(C))$.

The following result is useful for determining the covering radius of codes over rings. This is a generalization of the result in [13] for codes over finite fields.

Proposition 2. If $C_{0}$ and $C_{1}$ are codes over $\mathbb{R}$, of lengths $n_{0}$ and $n_{1}$, minimum distance $d_{0}$ and $d_{1}$, and generated by matrices $G_{0}$ and $G_{1}$, respectively, and if $C$ is the code generated by

$$
G=\left[\begin{array}{c|c}
0 & G_{1} \\
\hline G_{0} & A
\end{array}\right],
$$


then $r_{d}(C) \leq r_{d}\left(C_{0}\right)+r_{d}\left(C_{1}\right)$, and the covering radius of the concatenation of $C_{0}$ and $C_{1}$, denoted $C_{c}$, satisfies

$$
r_{d}\left(C_{c}\right) \geq r_{d}\left(C_{0}\right)+r_{d}\left(C_{1}\right)
$$

for all distances $d$ over $\mathbb{R}$.

\section{The covering radius of the block repetition codes over $\mathbb{R}$}

In order to determine the covering radius of simplex codes of types $\alpha$ and $\beta$ over $\mathbb{R}$, the some classes of block codes over $\mathbb{R}$ is defind and the approach in [14] is used to obtain the covering radius.

The block repetition code $C^{n}$ over $\mathbb{R}$ is a $\mathbb{R}$-additive code of length $n=\sum_{j=1}^{7} n_{j}$ with generator matrix $G=$ $(\overbrace{0101 \cdots 01}^{n_{1}} \overbrace{0 v 0 v \cdots 0 v}^{n_{2}} \overbrace{01+v 01+v \cdots 01+v}^{n_{3}} \overbrace{1010 \cdots 10}^{n_{4}} \overbrace{1111 \cdots 11}^{n_{5}} \overbrace{1 v 1 v \cdots 1 v}^{n_{6}} \overbrace{11+v 11+v \cdots 11+v}^{n_{7}})$.

If, for a fixed $1 \leq i \leq 7$. For all $1 \leq j \neq i \leq 7, n_{j}=0$, then the code $C^{n}=C^{n_{i}}$ is denoted by $C_{i}$. Therefore, the seven basic repetition codes are given below. That,

1. $C_{1}=\{(00 \cdots 00),(01 \cdots 01),(0 v \cdots 0 v),(01+v \cdots 01+v)\}$, is an additive code of length $n=n_{1}$ generated to $G_{1}=[0101 \cdots 01]$.

2. $C_{2}=\{(00 \cdots 00),(0 v \cdots 0 v)\}$, is an additive code of length $n=n_{2}$ generated to $G_{2}=[0 v 0 v \cdots 0 v]$.

3. $C_{3}=\{(00 \cdots 00),(01 \cdots 01),(0 v \cdots 0 v),(01+v \cdots 01+v)\}$, is an additive code of length $n=n_{3}$ generated to $G_{3}=[01+v 01+v \cdots 01+v]$,

4. $C_{4}=\{(00 \cdots 00),(10 \cdots 10)\}$, is an additive code of length $n=n_{4}$ generated to $G_{4}=[1010 \cdots 10]$,

5. $C_{5}=\{(00 \cdots 00),(01 \cdots 01),(0 v \cdots 0 v),(01+v \cdots 01+v),(10 \cdots 10),(11 \cdots 11)$,

$(1 v \cdots 1 v),(11+v \cdots 11+v)\}$, is an additive code of length $n=n_{5}$ generated to $G_{5}=[1111 \cdots 11]$,

6. $C_{6}=\{(00 \cdots 00),(0 v \cdots 0 v),(10 \cdots 10),(1 v \cdots 1 v)\}$, is an additive code of length $n=n_{6}$ generated to $G_{6}=[1 v 1 v \cdots 1 v]$,

7. $C_{7}=\{(00 \cdots 00),(01 \cdots 01),(0 v \cdots 0 v),(01+v \cdots 01+v),(10 \cdots 10),(11 \cdots 11)$,

$(1 v \cdots 1 v),(11+v \cdots 11+v)\}$, is an additive code of length $n=n_{7}$ generated to $G_{7}=$ $[11+v 11+v \cdots 11+v]$.

The following theorems provide the covering radius of $C_{j}$, for $1 \leq j \leq 7$.

Theorem 3. The covering radius of $C_{j}, 1 \leq j \leq 7$, with respect to the Euclidean weight is given by

1. $\frac{3 n}{4} \leq r_{E}\left(C_{1}\right)=r_{E}\left(C_{3}\right) \leq 2 n$

2. $n \leq r_{E}\left(C_{2}\right) \leq 3 n$

3. $\frac{n}{4} \leq r_{E}\left(C_{4}\right) \leq 4 n$,

4. $n \leq r_{E}\left(C_{5}\right)=r_{E}\left(C_{7}\right) \leq 2 n$,

5. $\frac{5 n}{4} \leq r_{E}\left(C_{6}\right) \leq \frac{5 n}{2}$.

Proof. For $c \in C_{j}, 1 \leq j \leq 7$, let $t_{i}(c), 0 \leq i \leq 7$ denote the number of occurrences of symbol $i$ in the codeword c. Considering 1 to 5 , that

$$
r_{E}\left(C_{j}\right)=\max _{x \in \mathbb{R}^{n}}\left\{d_{E}\left(x, C_{j}\right) ; 1 \leq j \leq 7\right\}
$$

Let $x \in \mathbb{R}^{n}$. If $x$ is given by $\left(t_{0}, t_{1}, t_{2}, t_{3}, t_{4}, t_{5}, t_{6}, t_{7}\right)$, where $\sum_{j=0}^{7} t_{j}=n$, then

$d_{E}(x, \overline{00})=n-t_{0}+3 t_{2}+t_{5}+4 t_{6}+t_{7}, d_{E}(x, \overline{01})=n-t_{1}+3 t_{3}+t_{4}+t_{6}+4 t_{7}$,

$d_{E}(x, \overline{0 v})=n-t_{2}+3 t_{0}+4 t_{4}+t_{5}+t_{7}, d_{E}(x, \overline{01+v})=n-t_{3}+3 t_{1}+t_{4}+4 t_{5}+t_{6}$,

$d_{E}(x, \overline{10})=n-t_{4}+t_{1}+4 t_{2}+t_{3}+3 t_{6}, d_{E}(x, \overline{11})=n-t_{5}+t_{0}+t_{2}+4 t_{3}+3 t_{7}$,

$d_{E}(x, \overline{1 v})=n-t_{6}+4 t_{0}+t_{1}+t_{3}+3 t_{4}, d_{E}(x, \overline{11+v})=n-t_{7}+t_{0}+4 t_{1}+t_{2}+3 t_{5}$.

Therefore, $d_{E}\left(x, C_{1}\right)=d_{E}\left(x, C_{3}\right)=\min \left\{\left(n-t_{0}+3 t_{2}+t_{5}+4 t_{6}+t_{7}\right),\left(n-t_{1}+3 t_{3}+t_{4}+t_{6}+4 t_{7}\right),(n-\right.$ $\left.\left.t_{2}+3 t_{0}+4 t_{4}+t_{5}+t_{7}\right),\left(n-t_{3}+3 t_{1}+t_{4}+4 t_{5}+t_{6}\right)\right\} \leq \frac{4 n+2\left(t_{0}+t_{1}+t_{2}+t_{3}+t_{4}+t_{5}+t_{6}+t_{7}\right)+2\left(t_{4}+t_{5}+t_{6}+t_{7}\right)}{4} \leq 2 n$ and hence

$$
r_{E}\left(C_{1}\right)=r_{E}\left(C_{3}\right) \leq 2 n
$$




$$
\begin{aligned}
& \text { If } x=(\overbrace{00 \cdots 00}^{\frac{n}{4}} \overbrace{01 \cdots 01}^{\frac{n}{4}} \overbrace{0 v \cdots 0 v}^{\frac{n}{4}} \overbrace{01+v \cdots 01+v}^{\frac{n}{4}}) \in \mathbb{R}^{n}, \text { then } \\
& d_{E}(x, \overline{00})=d_{E}(x, \overline{01})=d_{E}(x, \overline{0 u})=d_{E}(x, \overline{01+u})=\frac{n}{8}+4\left(\frac{n}{8}\right)+\frac{n}{8}=\frac{3 n}{4}
\end{aligned}
$$

and $r_{E}\left(C_{1}\right)=r_{E}\left(C_{3}\right) \geq \frac{3 n}{4}$. Thus, $\frac{3 n}{4} \leq r_{E}\left(C_{1}\right)=r_{E}\left(C_{3}\right) \leq 2 n$.

$$
\begin{aligned}
d_{E}\left(x, C_{2}\right) & =\min \left\{\left(n-t_{0}+3 t_{2}+t_{5}+4 t_{6}+t_{7}\right),\left(n-t_{2}+3 t_{0}+4 t_{4}+t_{5}+t_{7}\right)\right\} \\
& \leq \frac{2 n+2\left(t_{0}+t_{2}+t_{5}+t_{7}\right)+4\left(t_{4}+t_{6}\right)}{2} \leq 3 n
\end{aligned}
$$

Thus $r_{E}\left(C_{2}\right) \leq 3 n$.

$$
\begin{aligned}
\text { If } x=(\overbrace{00 \cdots 00}^{\frac{n}{2}} \overbrace{0 v \cdots 0 v}^{\frac{n}{2}}) \in \mathbb{R}^{n}, \text { then } \\
\qquad d_{E}(x, \overline{00})=d_{E}(x, \overline{0 v})=4\left(\frac{n}{4}\right)=n .
\end{aligned}
$$

Thus $r_{E}\left(C_{2}\right) \geq n$, and so, $n \leq r_{E}\left(C_{2}\right) \leq 3 n$.

$$
\begin{aligned}
d_{E}\left(x, C_{4}\right) & =\min \left\{\left(n-t_{0}+3 t_{2}+t_{5}+4 t_{6}+t_{7}\right),\left(n-t_{4}+t_{1}+4 t_{2}+t_{3}+3 t_{6}\right)\right\} \\
& \leq \frac{2 n-t_{0}+t_{1}+7 t_{2}+t_{3}-t_{4}+t_{5}+7 t_{6}+t_{7}}{2} \leq 4 n
\end{aligned}
$$

and hence $r_{E}\left(C_{4}\right) \leq 4 n$.

$$
\begin{aligned}
& \text { If } x=(\overbrace{00 \cdots 00}^{\frac{n}{2}} \overbrace{10 \cdots 10}^{\frac{n}{2}}) \in \mathbb{R}^{n} \text {, then } \\
& \qquad d_{E}(x, \overline{00})=d_{E}(x, \overline{10})=\frac{n}{4} .
\end{aligned}
$$

Thus $r_{E}\left(C_{4}\right) \geq \frac{n}{4}$, and so $\frac{n}{4} \leq r_{E}\left(C_{4}\right) \leq 4 n$.

$d_{E}\left(x, C_{5}\right)=d_{E}\left(x, C_{7}\right)=\min \left\{\left(n-t_{0}+3 t_{2}+t_{5}+4 t_{6}+t_{7}\right),\left(n-t_{1}+3 t_{3}+t_{4}+t_{6}+4 t_{7}\right),\left(n-t_{2}+3 t_{0}+4 t_{4}+\right.\right.$ $\left.t_{5}+t_{7}\right),\left(n-t_{3}+3 t_{1}+t_{4}+4 t_{5}+t_{6}\right),\left(n-t_{4}+t_{1}+4 t_{2}+t_{3}+3 t_{6}\right),\left(n-t_{5}+t_{0}+t_{2}+4 t_{3}+3 t_{7}\right),\left(n-t_{6}+4 t_{0}+\right.$ $\left.\left.t_{1}+t_{3}+3 t_{4}\right),\left(n-t_{7}+t_{0}+4 t_{1}+t_{2}+3 t_{5}\right)\right\} \leq 2 n$, then $r_{E}\left(C_{5}\right)=r_{E}\left(C_{7}\right) \leq 2 n$.

If $x=(\overbrace{00 \cdots 00}^{\frac{n}{8}} \overbrace{01 \cdots 01}^{\frac{n}{8}} \overbrace{0 v \cdots 0 v}^{\frac{n}{8}} \overbrace{01+v \cdots 01+v}^{\frac{n}{8}} \overbrace{10 \cdots 10}^{\frac{n}{8}} \overbrace{11 \cdots 11}^{\frac{n}{8}} \overbrace{1 v \cdots 1 v}^{\frac{n}{8}} \overbrace{11+v \cdots 1+v}^{\frac{n}{8}}) \in \mathbb{R}^{n}$, then $d_{E}(x, \overline{00})=d_{E}(x, \overline{01})=d_{E}(x, \overline{0 v})=d_{E}(x, \overline{01+v})=d_{E}(x, \overline{10})=d_{E}(x, \overline{11})=d_{E}(x, \overline{1 v})=d_{E}(x, \overline{11+v})=$ $\frac{n}{16}+4\left(\frac{n}{16}\right)+\frac{n}{16}+\frac{n}{16}+\frac{n}{8}+\frac{n}{16}+4\left(\frac{n}{16}\right)+\frac{n}{8}=n$.

Thus $r_{E}\left(C_{5}\right)=r_{E}\left(C_{7}\right) \geq n$, and so $n \leq r_{E}\left(C_{5}\right)=r_{E}\left(C_{7}\right) \leq 2 n$.

$d_{E}\left(x, C_{6}\right)=\min \left\{\left(n-t_{0}+3 t_{2}+t_{5}+4 t_{6}+t_{7}\right),\left(n-t_{2}+3 t_{0}+4 t_{4}+t_{5}+t_{7}\right),\left(n-t_{4}+t_{1}+4 t_{2}+t_{3}+\right.\right.$ $\left.\left.3 t_{6}\right),\left(n-t_{6}+4 t_{0}+t_{1}+t_{3}+3 t_{4}\right)\right\} \leq \frac{4 n+2 n+4 n}{4}$, so then $r_{E}\left(C_{6}\right) \leq \frac{5 n}{2}$.

If $x=(\overbrace{00 \cdots 00}^{\frac{n}{4}} \overbrace{0 v \cdots 0 v}^{\frac{n}{4}} \overbrace{10 \cdots 10}^{\frac{n}{4}} \overbrace{1 v \cdots 1 v}^{\frac{n}{4}}) \in \mathbb{R}^{n}$,

then

$d_{E}(x, \overline{00})=d_{E}(x, \overline{0 v})=d_{E}(x, \overline{10})=d_{E}(x, \overline{1 v})=4\left(\frac{n}{8}\right)+\frac{n}{8}+\frac{n}{8}+4\left(\frac{n}{8}\right)=\frac{5 n}{4}$.

Thus $r_{E}\left(C_{6}\right) \geq \frac{5 n}{4}$, and so $\frac{5 n}{4} \leq r_{E}\left(C_{6}\right) \leq \frac{5 n}{2}$. 
Theorem 4. The Covering radius of $C_{j}, 1 \leq j \leq 7$, with respect to the Lee weight is given by

1. $\frac{n}{2} \leq r_{L}\left(C_{1}\right)=r_{E}\left(C_{3}\right) \leq 2 n$,

2. $\frac{n}{2} \leq r_{L}\left(C_{2}\right) \leq 2 n$,

3. $\frac{n}{4} \leq r_{L}\left(C_{4}\right) \leq 2 n$,

4. $\frac{3 n}{4} \leq r_{L}\left(C_{5}\right)=r_{L}\left(C_{7}\right) \leq \frac{3 n}{2}$,

5. $\frac{3 n}{4} \leq r_{L}\left(C_{6}\right) \leq \frac{3 n}{2}$.

Proof. For $c \in C_{j}, 1 \leq j \leq 7$, let $t_{i}(c), 0 \leq i \leq 7$ denote the number of occurrences of symbol $i$ in the codeword c. Considering 1 to 5 , that

$$
r_{L}\left(C_{j}\right)=\max _{x \in \mathbb{R}^{n}}\left\{d_{L}\left(x, C_{j}\right) ; 1 \leq j \leq 7\right\}
$$

Let $x \in \mathbb{R}^{n}$. If $x$ is given by $\left(t_{0}, t_{1}, t_{2}, t_{3}, t_{4}, t_{5}, t_{6}, t_{7}\right)$, where $\sum_{j=0}^{7} t_{j}=n$, then

$d_{L}(x, \overline{00})=n-t_{0}+t_{2}+t_{5}+2 t_{6}+t_{7}, d_{L}(x, \overline{01})=n-t_{1}+t_{3}+t_{4}+t_{6}+2 t_{7}$,

$d_{L}(x, \overline{0 v})=n-t_{2}+t_{0}+2 t_{4}+t_{5}+t_{7}, d_{L}(x, \overline{01+v})=n-t_{3}+t_{1}+t_{4}+2 t_{5}+t_{6}$,

$d_{L}(x, \overline{10})=n-t_{4}+t_{1}+2 t_{2}+t_{3}+t_{6}, d_{L}(x, \overline{11})=n-t_{5}+t_{0}+t_{2}+2 t_{3}+t_{7}$,

$d_{L}(x, \overline{1 v})=n-t_{6}+2 t_{0}+t_{1}+t_{3}+t_{4}, d_{L}(x, \overline{11+v})=n-t_{7}+t_{0}+2 t_{1}+t_{2}+t_{5}$.

Therefore, $d_{L}\left(x, C_{1}\right)=d_{L}\left(x, C_{3}\right)=\min \left\{\left(n-t_{0}+t_{2}+t_{5}+2 t_{6}+t_{7}\right),\left(n-t_{1}+t_{3}+t_{4}+t_{6}+2 t_{7}\right),\left(n-t_{2}+t_{0}+\right.\right.$ $\left.\left.2 t_{4}+t_{5}+t_{7}\right),\left(n-t_{3}+t_{1}+t_{4}+2 t_{5}+t_{6}\right)\right\} \leq \frac{4 n+4\left(t_{4}+t_{5}+t_{6}+t_{7}\right)}{4} \leq 2 n$, then $r_{L}\left(C_{1}\right)=r_{L}\left(C_{3}\right) \leq 2 n$.

If $x=(\overbrace{00 \cdots 00}^{\frac{n}{4}} \overbrace{01 \cdots 01}^{\frac{n}{4}} \overbrace{0 v \cdots 0 v}^{\frac{n}{4}} \overbrace{01+v \cdots 01+v}^{\frac{n}{4}}) \in \mathbb{R}^{n}$, then $d_{L}(x, \overline{00})=d_{L}(x, \overline{01})=d_{L}(x, \overline{0 v})=$ $d_{L}(x, \overline{01+v})=\frac{n}{8}+2\left(\frac{n}{8}\right)+\frac{n}{8}=\frac{n}{2}$. Thus $r_{L}\left(C_{1}\right)=r_{L}\left(C_{3}\right) \geq \frac{n}{2}$, and so $\frac{n}{2} \leq r_{L}\left(C_{1}\right)=r_{L}\left(C_{3}\right) \leq 2 n$.

$d_{L}\left(x, C_{2}\right)=\min \left\{\left(n-t_{0}+t_{2}+t_{5}+2 t_{6}+t_{7}\right),\left(n-t_{2}+t_{0}+2 t_{4}+t_{5}+t_{7}\right)\right\} \leq \frac{2 n+2\left(t_{4}+t_{5}+t_{6}+t_{7}\right)}{2} \leq 2 n$. Then $r_{L}\left(C_{2}\right) \leq 2 n$.

If $x=(\overbrace{00 \cdots 00}^{\frac{n}{2}} \overbrace{0 v \cdots 0 v}^{\frac{n}{2}}) \in \mathbb{R}^{n}$, then $d_{L}(x, \overline{00})=d_{L}(x, \overline{0 v})=2\left(\frac{n}{4}\right)=\frac{n}{2}$. Thus $r_{L}\left(C_{2}\right) \geq \frac{n}{2}$ and so $\frac{n}{2} \leq r_{L}\left(C_{2}\right) \leq 2 n$

$d_{L}\left(x, C_{4}\right)=\min \left\{\left(n-t_{0}+t_{2}+t_{5}+2 t_{6}+t_{7}\right),\left(n-t_{4}+t_{1}+2 t_{2}+t_{3}+t_{6}\right)\right\} \leq \frac{2 n+2 n}{2} \leq 2 n$, so then $r_{L}\left(C_{4}\right) \leq 2 n$

If $x=(\overbrace{00 \cdots 00}^{\frac{n}{2}} \overbrace{10 \cdots 10}^{\frac{n}{2}}) \in \mathbb{R}^{n}$, then $d_{L}(x, \overline{00})=d_{L}(x, \overline{10})=\left(\frac{n}{4}\right)=\frac{n}{4}$. Thus $r_{L}\left(C_{4}\right) \geq \frac{n}{4}$ and hence $\frac{n}{4} \leq r_{L}\left(C_{4}\right) \leq 2 n$

$d_{L}\left(x, C_{5}\right)=d_{L}\left(x, C_{7}\right)=\min \left\{\left(n-t_{0}+t_{2}+t_{5}+2 t_{6}+t_{7}\right),\left(n-t_{1}+t_{3}+t_{4}+t_{6}+2 t_{7}\right),\left(n-t_{2}+t_{0}+2 t_{4}+\right.\right.$ $\left.t_{5}+t_{7}\right),\left(n-t_{3}+t_{1}+t_{4}+2 t_{5}+t_{6}\right),\left(n-t_{4}+t_{1}+2 t_{2}+t_{3}+t_{6}\right),\left(n-t_{5}+t_{0}+t_{2}+2 t_{3}+t_{7}\right),\left(n-t_{6}+2 t_{0}+\right.$ $\left.\left.t_{1}+t_{3}+t_{4}\right),\left(n-t_{7}+t_{0}+2 t_{1}+t_{2}+t_{5}\right)\right\} \leq \frac{8 n-n+5 n}{8} \leq \frac{3 n}{2}$, so then $r_{L}\left(C_{5}\right)=r_{L}\left(C_{7}\right) \leq \frac{3 n}{2}$.

If $x=(\overbrace{00 \cdots 00}^{\frac{n}{8}} \overbrace{01 \cdots 01}^{\frac{n}{8}} \overbrace{0 v \cdots 0 v}^{\frac{n}{8}} \overbrace{01+v \cdots 01+v}^{\frac{n}{8}} \overbrace{10 \cdots 10}^{\frac{n}{8}} \overbrace{11 \cdots 11}^{\frac{n}{8}} \overbrace{1 v \cdots 1 v}^{\frac{n}{8}} \overbrace{11+v \cdots 11+v}^{\frac{n}{8}}) \in \mathbb{R}^{n}$, then $d_{L}(x, \overline{00})=d_{L}(x, \overline{01})=d_{L}(x, \overline{0 v})=d_{L}(x, \overline{01+v})=d_{L}(x, \overline{10})=d_{L}(x, \overline{11})=d_{L}(x, \overline{1 v})=$ $d_{L}(x, \overline{11+v})=\frac{n}{16}+2\left(\frac{n}{16}\right)+\frac{n}{16}+\frac{n}{16}+\frac{n}{16}+\frac{n}{16}+\frac{n}{16}+2\left(\frac{n}{16}\right)+\frac{n}{16}+\frac{n}{16}=\frac{3 n}{4}$. Thus $r_{L}\left(C_{5}\right)=r_{L}\left(C_{7}\right) \geq \frac{3 n}{4}$, and so $\frac{3 n}{4} \leq r_{L}\left(C_{5}\right)=r_{L}\left(C_{7}\right) \leq \frac{3 n}{2} \cdot d_{L}\left(x, C_{6}\right)=\min \left\{\left(n-t_{0}+t_{2}+t_{5}+2 t_{6}+t_{7}\right),\left(n-t_{2}+t_{0}+2 t_{4}+t_{5}+\right.\right.$ $\left.\left.t_{7}\right),\left(n-t_{4}+t_{1}+2 t_{2}+t_{3}+t_{6}\right),\left(n-t_{6}+2 t_{0}+t_{1}+t_{3}+t_{4}\right)\right\} \leq \frac{4 n+2 n}{4} \leq \frac{3 n}{2}$, so then $r_{L}\left(C_{6}\right) \leq \frac{3 n}{2}$.

If $x=(\overbrace{00 \cdots 00}^{\frac{n}{4}} \overbrace{0 v \cdots 0 v}^{\frac{n}{4}} \overbrace{10 \cdots 10}^{\frac{n}{4}} \overbrace{1 v \cdots 1 v}^{\frac{n}{4}}) \in \mathbb{R}^{n}$, then $d_{L}(x, \overline{00})=d_{L}(x, \overline{0 v})=d_{L}(x, \overline{10})=d_{L}(x, \overline{1 v})=$ $2\left(\frac{n}{8}\right)+\frac{n}{8}+\frac{n}{8}+2\left(\frac{n}{8}\right)=\frac{3 n}{4}$. Thus $r_{L}\left(C_{6}\right) \geq \frac{3 n}{4}$, and hence $\frac{3 n}{4} \leq r_{L}\left(C_{6}\right) \leq \frac{3 n}{2}$.

Theorem 5. The covering radius of the block repetition code $C^{n}$ have the following properties; 


$$
r_{E}\left(C^{n}\right) \leq \frac{1}{2}\left[5\left(n_{1}+n_{3}+n_{6}\right)+3 n_{2}+9 n_{4}\right]+2\left(n_{5}+n_{7}\right)
$$

and if $n_{1}=\cdots=n_{7}=n$

$$
r_{L}\left(C^{7 n}\right)=2 n
$$

Proof. By Proposition 2, Theorem 3 and Theorem 4, let $x=x_{1} x_{2} x_{3} x_{4} x_{5} x_{6} x_{7} \in \mathbb{R}^{n}$ with $x_{1}, x_{2}, x_{3}, x_{4}, x_{5}, x_{6}, x_{7}$ is $\left(a_{0}, a_{1}, a_{2}, a_{3}, a_{4}, a_{5}, a_{6}, a_{7}\right),\left(b_{0}, b_{1}, b_{2}, b_{3}, b_{4}, b_{5}, b_{6}, b_{7}\right),\left(c_{0}, c_{1}, c_{2}, c_{3}, c_{4}, c_{5}, c_{6}, c_{7}\right),\left(d_{0}, d_{1}, d_{2}, d_{3}, d_{4}, d_{5}, d_{6}, d_{7}\right)$, $\left(e_{0}, e_{1}, e_{2}, e_{3}, e_{4}, e_{5}, e_{6}, e_{7}\right),\left(f_{0}, f_{1}, f_{2}, f_{3}, f_{4}, f_{5}, f_{6}, f_{7}\right),\left(g_{0}, g_{1}, g_{2}, g_{3}, g_{4}, g_{5}, g_{6}, g_{7}\right)$, respectively such that $n_{1}=$ $\sum_{j=0}^{7} a_{j}, n_{2}=\sum_{j=0}^{7} b_{j}, n_{3}=\sum_{j=0}^{7} c_{j}, n_{4}=\sum_{j=0}^{7} d_{j}, n_{5}=\sum_{j=0}^{7} e_{j}, n_{6}=\sum_{j=0}^{7} f_{j}, n_{7}=\sum_{j=0}^{7} g_{j}$. Then $d_{E}(x, \overline{00})=$ $n_{1}-a_{0}+3 a_{2}+a_{5}+4 a_{6}+a_{7}+n_{2}-b_{0}+3 b_{2}+b_{5}+4 b_{6}+b_{7}+n_{3}-c_{0}+3 c_{2}+c_{5}+4 c_{6}+c_{7}+n_{4}-d_{0}+3 d_{2}+$ $d_{5}+4 d_{6}+d_{7}+n_{5}-e_{0}+3 e_{2}+e_{5}+4 e_{6}+e_{7}+n_{6}-f_{0}+3 f_{2}+f_{5}+4 f_{6}+f_{7}+n_{7}-g_{0}+3 g_{2}+g_{5}+4 g_{6}+g_{7}$ where $\overline{00}=\overbrace{00 \cdots 00}^{n_{1}} \overbrace{00 \cdots 00}^{n_{2}} \overbrace{00 \cdots 00}^{n_{3}} \overbrace{00 \cdots 00}^{n_{4}} \overbrace{00 \cdots 00}^{n_{0}} \overbrace{00 \cdots 00}^{n_{5}} \overbrace{00 \cdots 00}^{n_{6}}$, is the first vector of $C^{n}$, where $n=n_{1}$. $d_{E}\left(x, \overline{y_{1}}\right)=n_{1}-a_{1}+3 a_{3}+a_{4}+a_{6}+4 a_{7}+n_{2}-b_{2}+3 b_{0}+4 b_{4}+b_{5}+b_{7}+n_{3}-c_{3}+3 c_{1}+c_{4}+4 c_{5}+c_{6}+$ $n_{4}-d_{4}+d_{1}+4 d_{2}+d_{3}+3 d_{6}+n_{5}-e_{5}+e_{0}+e_{2}+4 e_{3}+3 e_{7}+n_{6}-f_{6}+4 f_{0}+f_{1}+f_{3}+3 f_{4}+n_{7}-g_{7}+g_{0}+$ $4 g_{1}+g_{2}+3 g_{5}$, where $\overline{y_{1}}=\overbrace{01 \cdots 01}^{n_{1}} \overbrace{0 v \cdots 0 v}^{n_{2}} \overbrace{01+v \cdots 01+v}^{n_{3}} \overbrace{10 \cdots 10}^{n_{4}} \overbrace{11 \cdots 11}^{n_{5}} \overbrace{1 v \cdots 1 v}^{n_{6}} \overbrace{11+v \cdots 11+v}^{n_{1}}$, is the second vector of $C^{n}$, where $n=n_{2}$.

$d_{E}\left(x, \overline{y_{2}}\right)=n_{1}-a_{1}+3 a_{3}+a_{4}+a_{6}+4 a_{7}+n_{2}-b_{2}+3 b_{0}+4 b_{4}+b_{5}+b_{7}+n_{3}-c_{3}+3 c_{1}+c_{4}+4 c_{5}+c_{6}+$ $n_{4}-d_{0}+3 d_{2}+d_{5}+4 d_{6}+d_{7}+n_{5}-e_{1}+3 e_{3}+e_{4}+e_{6}+4 e_{7}+n_{6}-f_{2}+3 f_{0}+4 f_{4}+f_{5}+f_{7}+n_{7}-g_{3}+3 g_{1}+$ $g_{4}+4 g_{5}+g_{6}$, where $\overline{y_{2}}=\overbrace{01 \cdots 01}^{n_{1}} \overbrace{0 v \cdots 0 v}^{n_{2}} \overbrace{01+v \cdots 01+v}^{n_{3}} \overbrace{00 \cdots 00}^{n_{4}} \overbrace{01 \cdots 01}^{n_{5}} \overbrace{0 v \cdots 0 v}^{n_{0}} \overbrace{01+v \cdots 01+v}^{n_{6}}$, is the third vector of $C^{n}$, where $n=n_{3}$.

$d_{E}\left(x, \overline{y_{3}}\right)=n_{1}-a_{2}+3 a_{0}+4 a_{4}+a_{5}+a_{7}+n_{2}-b_{0}+3 b_{2}+b_{5}+4 b_{6}+b_{7}+n_{3}-c_{2}+3 c_{0}+4 c_{4}+c_{5}+c_{7}+$ $n_{4}-d_{0}+3 d_{2}+d_{5}+4 d_{6}+d_{7}+n_{5}-e_{2}+3 e_{0}+4 e_{4}+e_{5}+e_{7}+n_{6}-f_{0}+3 f_{2}+f_{5}+4 f_{6}+f_{7}+n_{7}-g_{2}+3 g_{0}+$ $4 g_{4}+g_{5}+g_{7}$, where $\overline{y_{3}}=\overbrace{0 v \cdots 0 v}^{n_{1}} \overbrace{00 \cdots 00}^{n_{2}} \overbrace{0 v \cdots 0 v}^{n_{3}} \overbrace{00 \cdots 00}^{n_{4}} \overbrace{0 v \cdots 0 v}^{n_{5}} \overbrace{00 \cdots 00}^{n_{0}} \overbrace{0 v \cdots 0 v}^{n_{6}} \overbrace{7}$, is the fourth vector of $C^{n}$, where $n=n_{4}$.

$d_{E}\left(x, \overline{y_{4}}\right)=n_{1}-a_{3}+3 a_{1}+a_{4}+4 a_{5}+a_{6}+n_{2}-b_{2}+3 b_{0}+4 b_{4}+b_{5}+b_{7}+n_{3}-c_{1}+3 c_{3}+c_{4}+c_{6}+4 c_{7}+$ $n_{4}-d_{0}+3 d_{2}+d_{5}+4 d_{6}+d_{7}+n_{5}-e_{3}+3 e_{1}+e_{4}+4 e_{5}+e_{6}+n_{6}-f_{2}+3 f_{0}+4 f_{4}+f_{5}+f_{7}+n_{7}-g_{1}+3 g_{3}+$ $g_{4}+g_{6}+4 g_{7}$, where $\overline{y_{4}}=\overbrace{01+v \cdots 01+v}^{n_{1}} \overbrace{0 v \cdots 0 v}^{n_{2}} \overbrace{01 \cdots 01}^{n_{3}} \overbrace{00 \cdots 00}^{n_{4}} \overbrace{01+v \cdots 01+v}^{n_{0}} \overbrace{0 v \cdots 0 v}^{n_{6}}$

$\overbrace{01+v \cdots 01+v}^{n_{7}}$, is the fifth vector of $C^{n}$, where $n=n_{5}$.

$d_{E}\left(x, \overline{y_{5}}\right)=n_{1}-a_{0}+3 a_{2}+a_{5}+4 a_{6}+a_{7}+n_{2}-b_{0}+3 b_{2}+b_{5}+4 b_{6}+b_{7}+n_{3}-c_{0}+3 c_{2}+c_{5}+4 c_{6}+c_{7}+$ $n_{4}-d_{4}+d_{1}+4 d_{2}+d_{3}+3 d_{6}+n_{5}-e_{4}+e_{1}+4 e_{2}+e_{3}+3 e_{6}+n_{6}-f_{4}+f_{1}+4 f_{2}+f_{3}+3 f_{6}+n_{7}-g_{4}+g_{1}+$ $4 g_{2}+g_{3}+3 g_{6}$, where $\overline{y_{5}}=\overbrace{00 \cdots 00}^{n_{1}} \overbrace{00 \cdots 00}^{n_{2}} \overbrace{00 \cdots 00}^{n_{3}} \overbrace{10 \cdots 10}^{n_{4}} \overbrace{10 \cdots 10}^{n_{5}} \overbrace{10 \cdots 10}^{n_{6}} \overbrace{10 \cdots 10}^{n_{7}}$, is the sixth vector of $C^{n}$, where $n=n_{6}$.

$d_{E}\left(x, \overline{y_{6}}\right)=n_{1}-a_{2}+3 a_{0}+4 a_{4}+a_{5}+a_{7}+n_{2}-b_{0}+3 b_{2}+b_{5}+4 b_{6}+b_{7}+n_{3}-c_{2}+3 c_{0}+4 c_{4}+c_{5}+c_{7}+$ $n_{4}-d_{4}+d_{1}+4 d_{2}+d_{3}+3 d_{6}+n_{5}-e_{6}+4 e_{0}+e_{1}+e_{3}+3 e_{4}+n_{6}-f_{4}+f_{1}+4 f_{2}+f_{3}+3 f_{6}+n_{7}-g_{6}+4 g_{0}+$ $g_{1}+g_{3}+3 g_{4}$, where $\overline{y_{6}}=\overbrace{0 v \cdots 0 v}^{n_{1}} \overbrace{00 \cdots 00}^{n_{2}} \overbrace{0 v \cdots 0 v}^{n_{3}} \overbrace{10 \cdots 10}^{n_{4}} \overbrace{1 v \cdots 1 v}^{n_{1}} \overbrace{10 \cdots 10}^{n_{10} n_{10}} \overbrace{1 v}^{n_{7}}$, is the seventh vector of $C^{n}$, where $n=n_{7}$.

$d_{E}\left(x, \overline{y_{7}}\right)=n_{1}-a_{3}+3 a_{1}+a_{4}+4 a_{5}+a_{6}+n_{2}-b_{2}+3 b_{0}+4 b_{4}+b_{5}+b_{7}+n_{3}-c_{1}+3 c_{3}+c_{4}+c_{6}+4 c_{7}+$ $n_{4}-d_{4}+d_{1}+4 d_{2}+d_{3}+3 d_{6}+n_{5}-e_{7}+e_{0}+4 e_{1}+e_{2}+3 e_{5}+n_{6}-f_{6}+4 f_{0}+f_{1}+f_{3}+3 f_{4}+n_{7}-g_{5}+g_{0}+$ $g_{2}+4 g_{3}+3 g_{7}$, where $\overline{y_{7}}=\overbrace{01+v \cdots 01+v}^{n_{0 v \cdots 0 v}} \overbrace{01 \cdots 01}^{n_{1}} \overbrace{10 \cdots 10}^{n_{1}} \overbrace{11+v \cdots 11+v}^{n_{1}} \overbrace{1 v \cdots 1 v}^{n_{1}} \overbrace{11 \cdots 11}^{n_{2}}$, is the eighth vector of $C^{n}$, where $n=n_{8}$. 
Thus

$$
\begin{aligned}
& r_{E}\left(C^{n}\right) \leq \frac{8 n_{1}+4\left(a_{0}+a_{1}+a_{2}+a_{3}\right)+12\left(a_{4}+a_{5}+a_{6}+a_{7}\right)+8 n_{2}+8\left(b_{0}+b_{2}+b_{5}+b_{7}\right)+16\left(b_{4}+b_{6}\right)}{8} \\
& +\frac{8 n_{3}+4\left(c_{0}+c_{1}+c_{2}+c_{3}\right)+12\left(c_{4}+c_{5}+c_{6}+c_{7}\right)+8 n_{4}-4\left(d_{0}+d_{4}\right)+4\left(d_{1}+d_{3}+d_{5}+d_{7}\right)+28\left(d_{2}+d_{6}\right)}{8} \\
& +\frac{8 n_{5}+8\left(e_{0}+e_{1}+e_{2}+e_{3}+e_{4}+e_{5}+e_{6}+e_{7}\right)+8 n_{6}+12\left(f_{0}+f_{2}+f_{4}+f_{6}\right)+4\left(f_{1}+f_{3}+f_{5}+f_{7}\right)}{8} \\
& +\frac{8 n_{7}+8\left(g_{0}+g_{1}+g_{2}+g_{3}+g_{4}+g_{5}+g_{6}+g_{7}\right)}{8} .
\end{aligned}
$$

Hence

$$
r_{E}\left(C^{n}\right) \leq \frac{1}{2}\left[5\left(n_{1}+n_{3}+n_{6}\right)+3 n_{2}+9 n_{4}\right]+2\left(n_{5}+n_{7}\right) .
$$

For the second part, that $\phi\left(C^{7 n}\right)$ is the set given by

$\{000 \cdots 000000 \cdots 000000 \cdots 000000 \cdots 000000 \cdots 000000 \cdots 000000 \cdots 000$,

$001 \cdots 001011 \cdots 011010 \cdots 010100 \cdots 100101 \cdots 101111 \cdots 111110 \cdots 110$,

$001 \cdots 001011 \cdots 011010 \cdots 010000 \cdots 000001 \cdots 001011 \cdots 011010 \cdots 010$,

$011 \cdots 011000 \cdots 000011 \cdots 011000 \cdots 000011 \cdots 011000 \cdots 000011 \cdots 011$,

$010 \cdots 010011 \cdots 011001 \cdots 001000 \cdots 000010 \cdots 010011 \cdots 011001 \cdots 001$,

$000 \cdots 000000 \cdots 000000 \cdots 000100 \cdots 100100 \cdots 100100 \cdots 100100 \cdots 100$,

$011 \cdots 011000 \cdots 000011 \cdots 011100 \cdots 100111 \cdots 111100 \cdots 100111 \cdots 111$,

$010 \cdots 010011 \cdots 011001 \cdots 001100 \cdots 100110 \cdots 110111 \cdots 111101 \cdots 101\}$.

Then $r_{L}\left(c^{7 n}\right)=r\left(\phi\left(C^{7 n}\right)\right)=2 n$.

\section{Simplex codes of types $\alpha$ and $\beta$}

In this section, consider the construction of simplex codes of types $\alpha$ and $\beta$ over $\mathbb{R}$. Let $m_{2, k}^{\alpha}$ be the generator matrix of $S_{2, k}^{\alpha}$ the binary simplex code of type $\alpha$, defined as

$$
\left[\begin{array}{c|c}
00 \cdots 0 & 11 \cdots 1 \\
\hline m_{2, k-1}^{\alpha} & m_{2, k-1}^{\alpha}
\end{array}\right], \text { for } k \geq 2,
$$

where

$$
m_{2,1}^{\alpha}=[0,1]
$$

In [8], the simplex codes $S_{4, k}^{\alpha}$ of type $\alpha$ over $R^{*}$ were defined. The generator matrix $G_{R^{*}, k}^{\alpha}$ of $S_{R^{*}, k}^{\alpha}$ is

$$
\left[\begin{array}{c|c|c|c}
00 \cdots 0 & 11 \cdots 1 & v v \cdots v & 1+v 1+v \cdots 1+v \\
\hline G_{R^{*}, k-1}^{\alpha} & G_{R^{*}, k-1}^{\alpha} & G_{R^{*}, k-1}^{\alpha} & G_{R^{*}, k-1}^{\alpha}
\end{array}\right], \text { for } k \geq 2,
$$

where

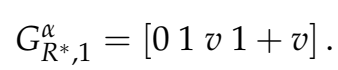

The generator matrix of $S_{k}^{\alpha}$, the simplex code of type $\alpha$ over $\mathbb{R}$ is dfined, as the concatenation of $2^{2 k}$ copies of the generator matrix of $S_{2, k}^{\alpha}$ and $2^{k}$ copies of the generator matrix of $S_{R^{*}, k}^{\alpha}$ given by

$$
\begin{gathered}
\Theta_{k}^{\alpha}=\left[m_{2, k}^{\alpha}\left|m_{2, k}^{\alpha}\right| \cdots\left|m_{2, k}^{\alpha}\right| G_{R^{*}, k}^{\alpha}\left|G_{R^{*}, k}^{\alpha}\right| \cdots \mid G_{R^{*}, k}^{\alpha}\right], \\
\text { for } k \geq 1 .
\end{gathered}
$$

The standard form of $\Theta_{k}^{\alpha}$ of the generator matrix of $S_{k}^{\alpha}$ is given by

$$
\Theta_{k}^{\alpha}=\left[\begin{array}{c|c|c|c}
0000 \cdots 00 & 0101 \cdots 01 & \cdots & 11+v 11+v \cdots 11+v \\
\hline \Theta_{k-1}^{\alpha} & \Theta_{k-1}^{\alpha} & \cdots & \Theta_{k-1}^{\alpha}
\end{array}\right], \text { for } k \geq 2,
$$


where

$$
\Theta_{1}^{\alpha}=[00010 v 01+v 10111 v 11+v]
$$

The length of the simplex code of type $\alpha$ over $\mathbb{R}$ is equal to $2^{3 k+1}$, and the number of codewords is equal to $2^{k_{0}} R^{* k_{1}}$ for some $k_{0}$ and $k_{1}$. In the case where $k=1$ with $k_{0}=0$ and $k_{1}=1$, that all of the codewords of the simplex code $S_{1}^{\alpha}$ are generated by $\Theta_{1}^{\alpha}$ and are given by

$$
\begin{gathered}
0000000000000000 \\
00010 v 01+v 10111 v 11+v \\
000 v 000 v 000 v 000 v \\
0001+v 0 v 011011+v 1 v 11
\end{gathered}
$$

The type $\beta$ simplex code $S_{k}^{\beta}$ is a punctured version of $S_{k}^{\alpha}$. The number of codewords is $2^{k_{0}} R^{* k_{1}}$ for some $k_{0}$ and $k_{1}$ and its length is $2^{k}\left(2^{k-2}+1\right)\left(2^{k}-1\right)$.

The generator matrix of $S_{k}^{\beta}$ is the concatenation of $2^{k}$ copies of the generator matrix of $S_{2, k}^{\beta}$ and $2^{k-1}$ copies of the generator matrix of $S_{R^{*}, k}^{\beta}$ given by

$$
\Theta_{k}^{\beta}=\left[m_{2, k}^{\beta}\left|m_{2, k}^{\beta}\right| \cdots\left|m_{2, k}^{\beta}\right| G_{R^{*}, k}^{\beta}\left|G_{R^{*}, k}^{\beta}\right| \cdots \mid G_{R^{*}, k}^{\beta}\right], \text { for } k \geq 2,
$$

where $m_{2, k}^{\beta}$ is the generator matrix of the binary simplex code of type $\beta$ is given by

$$
\left[\begin{array}{c|c}
11 \cdots 1 & 00 \cdots 0 \\
\hline m_{2, k-1}^{\alpha} & m_{2, k-1}^{\beta}
\end{array}\right], \text { for } k \geq 3,
$$

with

$$
m_{2,2}^{\beta}=\left[\begin{array}{l|l}
11 & 0 \\
\hline 01 & 1
\end{array}\right],
$$

and $G_{R^{*}, k}^{\beta}$ is a generator matrix of the simplex code over $R^{*}$ of type $\beta$ defined as

$$
\left[\begin{array}{c|c|c}
11 \cdots 1 & 00 \cdots 0 & v v \cdots v \\
\hline G_{R^{*}, k-1}^{\alpha} & G_{R^{*}, k-1}^{\beta} & G_{R^{*}, k-1}^{\beta}
\end{array}\right], \text { for } k \geq 3,
$$

with

$$
G_{R^{*}, 2}^{\beta}=\left[\begin{array}{c|c|c}
1111 & 0 & v \\
\hline 01 v 1+v & 1 & 1
\end{array}\right] .
$$

\subsection{The Covering radius of the simplex codes of types $\alpha$ and $\beta$}

The following theorems provide upper bounds on the covering radius of simplex codes over $\mathbb{R}$ with respect to the Lee and Euclidean weights.

Theorem 6 . The covering radius of the $\mathbb{R}$-simplex codes of type $\alpha$ are upper bounded as follows:

$$
r_{L}\left(S_{k}^{\alpha}\right) \leq 3.2^{3 k-1} \text { and } r_{E}\left(S_{k}^{\alpha}\right) \leq 2^{k}\left(\frac{7.2^{2 k}-1}{3}\right) .
$$


Proof. In $\mathbb{R}$-Simplex codes of type $\alpha$ have a Lee weight equal to $2^{3 k}$ or $3.2^{3 k-1}$. Hence, from (2), Proposition 2 and Theorem 5, we have

$$
\begin{aligned}
r_{L}\left(S_{k}^{\alpha}\right) & \leq r_{L}\left(2^{2 k} S_{2, k}^{\alpha}\right)+r_{L}\left(2^{k} S_{R^{*}, k}^{\alpha}\right) \\
& \leq 2^{2 k} r_{L}\left(S_{2, k}^{\alpha}\right)+2^{k} r_{L}\left(S_{R^{*}, k}^{\alpha}\right) \\
& \leq 2^{2 k} r_{H}\left(S_{2, k}^{\alpha}\right)+2^{k} r_{L}\left(S_{R^{*}, k}^{\alpha}\right) \\
& \leq 2^{2 k}\left(2^{k-1}\right)+2^{k}\left[\left(3 \cdot 2^{2(k-1)}+3 \cdot 2^{2(k-2)}+\cdots+3.2^{2 \cdot 1}\right)+r_{L}\left(S_{R^{*}, 1}^{\alpha}\right)\right] \\
& \leq 2^{3 k-1}+2^{k}\left[\left(2^{2 k}-1\right)+1\right] \\
& \leq 2^{3 k-1}+2^{k} \cdot 2^{2 k} \\
& \leq 2^{3 k-1}+2^{3 k} \leq 3.2^{3 k-1} .
\end{aligned}
$$

Thus $r_{L}\left(S_{k}^{\alpha}\right) \leq 3.2^{3 k-1}$. Similar arguments using (2), Proposition 2 and Theorem 5 give that

$$
\begin{aligned}
r_{E}\left(S_{k}^{\alpha}\right) & \leq r_{E}\left(2^{2 k} S_{2, k}^{\alpha}\right)+r_{E}\left(2^{k} S_{R^{*}, k}^{\alpha}\right) \\
& \leq 2^{2 k} r_{E}\left(S_{2, k}^{\alpha}\right)+2^{k} r_{E}\left(S_{R^{*}, k}^{\alpha}\right) \\
& \leq 2^{2 k} r_{H}\left(S_{2, k}^{\alpha}\right)+2^{k} r_{E}\left(S_{R^{*}, k}^{\alpha}\right) \\
& \leq 2^{2 k} \cdot 2^{k-1}+2^{k}\left(\frac{11\left(2^{2 k}-1\right)+9}{6}\right) \\
& \leq 2^{k}\left[2^{2 k-1}+\left(\frac{11\left(2^{2 k}-1\right)+9}{6}\right)\right] \\
& \leq 2^{k}\left(\frac{7.2^{2 k}-1}{3}\right) .
\end{aligned}
$$

Theorem 7. The covering radius of the $\mathbb{R}$-Simplex codes of type $\beta$ are given by

(i) $r_{L}\left(S_{k}^{\beta}\right) \leq 2^{k-1}\left[\left(2^{k-1}+1\right)\left(2^{k}-1\right)-2\right]$,

(ii) $r_{E}\left(S_{k}^{\beta}\right) \leq 2^{k-1}\left(\frac{14.2^{2 k}-449}{6}\right)$.

Proof. From (1), (3), Proposition 2 and Theorem 5, we have

$$
\begin{aligned}
r_{L}\left(S_{k}^{\beta}\right) & \leq r_{L}\left(2^{k} S_{2, k}^{\beta}\right)+r_{L}\left(2^{k-1} S_{R^{*}, k}^{\beta}\right) \\
& \leq 2^{k} r_{L}\left(S_{2, k}^{\beta}\right)+2^{k-1} r_{L}\left(S_{R^{*}, k}^{\beta}\right) \\
& \leq 2^{k} r_{H}\left(S_{2, k}^{\beta}\right)+2^{k-1} r_{L}\left(S_{R^{*}, k}^{\beta}\right) \\
& \leq 2^{k}\left(\frac{2^{k}-1}{)} 2+2^{k-1}\left[2^{k-1}\left(2^{k}-1\right)-2\right]\right. \\
& \leq 2^{k-1}\left(2^{k}-1\right)+2^{k-1}\left[2^{k-1}\left(2^{k}-1\right)-2\right] \\
& \leq 2^{k-1}\left[\left(2^{k-1}+1\right)\left(2^{k}-1\right)-2\right] .
\end{aligned}
$$


Similar arguments using (2), Proposition 2 and Theorem 5 give that

$$
\begin{aligned}
r_{E}\left(S_{k}^{\beta}\right) & \leq r_{E}\left(2^{k} S_{2, k}^{\beta}\right)+r_{E}\left(2^{k-1} S_{R^{*}, k}^{\beta}\right) \\
& \leq 2^{k} r_{E}\left(S_{2, k}^{\beta}\right)+2^{k-1} r_{E}\left(S_{R^{*}, k}^{\beta}\right) \\
& \leq 2^{k} r_{H}\left(S_{2, k}^{\beta}\right)+2^{k-1} r_{E}\left(S_{R^{*}, k}^{\beta}\right) \\
& \leq 2^{k}\left(\frac{2^{k}-1}{2}\right)+2^{k-1}\left[2^{k}\left(2^{k+1}-1\right)+\frac{1}{3}\left(2^{2 k}-1\right)-\frac{147}{2}\right]\left(\operatorname{sincer}_{E}\left(S_{2}^{\beta}\right) \leq 25\right) \\
& \leq 2^{k-1}\left(2^{k}-1\right)+2^{k-1}\left[2^{k}\left(2^{k+1}-1\right)+\frac{1}{3}\left(2^{2 k}-1\right)-\frac{147}{2}\right] \\
& \leq 2^{k-1}\left[2^{k}\left(2^{k+1}-1\right)+\frac{1}{3}\left(2^{2 k}-1\right)+\left(2^{k}-1\right)-\frac{147}{2}\right] \\
& \leq 2^{k-1}\left(\frac{14.2^{2 k}-449}{6}\right) .
\end{aligned}
$$

Conflicts of Interest: "The author declare no conflict of interest."

\section{References}

[1] Delsarte, P. (1973). An algebraic approach to the association schemes of coding theory, Philips Research Rep. Suppl. 10.

[2] Delsarte, P., \& Levenshtein, V. I. (1998). Association schemes and coding theory. IEEE Transactions on Information Theory, 44(6), 2477-2504.

[3] Cohen, G., Karpovsky, M., Mattson, H., \& Schatz, J. (1985). Covering radius-Survey and recent results. IEEE Transactions on Information Theory, 31(3), 328-343.

[4] Abualrub, T., Siap, I. \& Aydin, N. (2014). $\mathbb{Z}_{2} Z_{4}$ - additive cyclic codes. IEEE Transactions on Information Theory, 60(3), $115-121$.

[5] Bilal, M., Borges, J., Dougherty, S.T., \& Fernández-Córdoba, C. (2011). Maximum distance separable codes over $\mathbb{Z}_{4}$ and $\mathbb{Z}_{2} Z_{4}$. Des. Codes Cryptogr.,61(1), 31-40.

[6] Borges, J., Dougherty, S. T., \& Fernández-Córdoba, C. (2012). Characterization and constructions of self-dual codes over $\mathbb{Z}_{2} Z_{4}$. Adv. Math. Commun.,6(3), 287-303.

[7] Borges, J., Fernández-Córdoba, C., Pujol, J., Rifà, J., \& Villanueva, M. (2010). $\mathbb{Z}_{2} Z_{4}$-linear codes: generator matrices and duality. Designs, Codes and Cryptography, 54(2), 167-179.

[8] Bhandari, M. C., Gupta, M. K., \& Lal, A. K. (1999, November). On $\mathbb{Z}_{4}$-Simplex codes and their gray images. In International Symposium on Applied Algebra, Algebraic Algorithms, and Error-Correcting Codes (pp. 170-179). Springer, Berlin, Heidelberg.

[9] Gupta, M.K. (1999). On some linear codes over $\mathbb{Z}_{2^{s}}$. Ph.D. Thesis, IIT Kanpur.

[10] Gupta, M., \& Durairajan, C. (2012). On the covering radius of some modular codes. Advances in Mathematics of Communications,8(2), 129-137.

[11] ChellaPandian P., \& Durairajan, C. (2014). On the Covering radius of some Codes over $R=\mathbb{Z}_{2}+u \mathbb{Z}_{2}$ where $u^{2}=0$. International Journal of Research in Applied, Natural and Social Sciences,2(1), 61-70.

[12] Aoki, T., Gaborit, P., Harada, M., Ozeki, M., \& Sole, P. (1999). On the covering radius of $\mathbb{Z}_{4}$-codes and their lattices. IEEE Transactions on Information Theory, 45(6), 2162-2168.

[13] Cohen, G., Karpovsky, M., Mattson, H., \& Schatz, J. (1985). Covering radius-Survey and recent results. IEEE Transactions on Information Theory, 31(3), 328-343.

[14] Raut, M. K., \& Gupta, M. K. (2014). On octonary codes and their covering radii. Australasian Journal of Combinatorics, 63(2), 246-261. 